\title{
IRRADIATION LABORATORY OF THE BRITISH INSULATED CALLENDER'S CABLES, LTD., RESEARCH ORGANIZATION
}

\author{
By DR. R. M. BLACK
}

\begin{abstract}
T $\mathrm{T}$ is now fairly well known that, when polymeric materials are exposed to high-energy radiation in atomic piles or from sources of beta- or gammaradiation, they undergo profound changes in their physical properties. These changes are the result of a series of intermolecular reactions or rearrangements induced by the ionization and free-radical formation phenomena associated with the absorption of the radiation.
\end{abstract}

A study of the effects of irradiation of vinyl poly. mers and related hydrocarbons has shown that the radiation chemistry of the process involves a number of fundamental chemical changes : $(a)$ formation of carbon-carbon bonds between the molecules accompanied by the liberation of hydrogen to give a threedimensional polymer network; (b) formation and hydrogenation of carbon-carbon double bonds; (c) molecular degradation by fracture of the main polymer chain by a disproportionation reaction resulting in a product of lower average molecular weight; and $(d)$ fracture of chemical bonds in the side-chains or branches of the polymer, with the production of low molecular weight hydrocarbons.

The balance between these reactions is not the same in all polymers and, in practice, it is found that either cross-linking or molecular degradation predominates, depending upon the molecular structure. Thus, dielectric materials can be classified into two categories : those in which cross-linking predominates (such as polyethylene, polystyrene, polyvinyl chloride and the silicones) and those in which degradation predominates (such as polyisobutylene, 'Perspex', polytetrafluoroethylene and cellulose). Some materials, by virtue of their chemical structure and the possibility of energy transfer and resonance effects, are more resistant to the effects of radiation than others. Polymers which contain aromatic side-chains (phenyl groups) are particularly resistant to radiation.

The behaviour of polymeric dielectric materials on irradiation is of interest for two main reasons. First, it may prove possible, by irradiation, to induce changes in the structure of the material which cannot be achieved by other methods and which will result in a product with improved properties. Second, if the material is to be used in a locality in which it will be subjected to constant irradiation, such as the biological shield of a nuclear reactor, the expected service life and a knowledge of the changes in electrical properties likely to take place are important considerations.

In order to investigate and, if possible, to exploit the properties produced in dielectric materials on irradiation, the Research Organization of British Insulated Callender's Cables, Ltd., has recently established an irradiation laboratory. This laboratory is equipped with a 2-million volt Van de Graaff electrostatic particle accelerator supplied by the High Voltage Engineering Corporation of Cambridge, Massachusetts, which serves as a versatile source of electrons, gamma- or X-rays, positive ions and neutrons. In addition, there is available a compre- hensive radiochemical laboratory, equipped with a wide range of counting equipment and radioactive isotope sources, for the development of new techniques for testing and process control.

The Irradiation Laboratory is divided roughly into three parts by the central, two-story, radiation block. 'The latter consists of a target room on the ground floor with the generator room situated immediately above it. The walls of the target room are of concrete, $3 \mathrm{ft}$. in thickness, to prevent hazardous amounts of radiation entering the adjoining laboratories, and entrance to the room is by means of a safety door, connected to an interlock system, and a concrete labyrinth.

The target room is of sufficient size to enable a variety of irradiation experiments involving the use of conveyor belts and pulley systems, etc., to be carried out and, in addition, houses the pressurizing gas supply (carbon dioxide and nitrogen) for the generator.

The generator itself, which is designed to project a beam of electrons or positive ions vertically downwards, is installed in the room above, access to which is obtained by means of a spiral stairway from the target room. As the radiation intensity is consider-

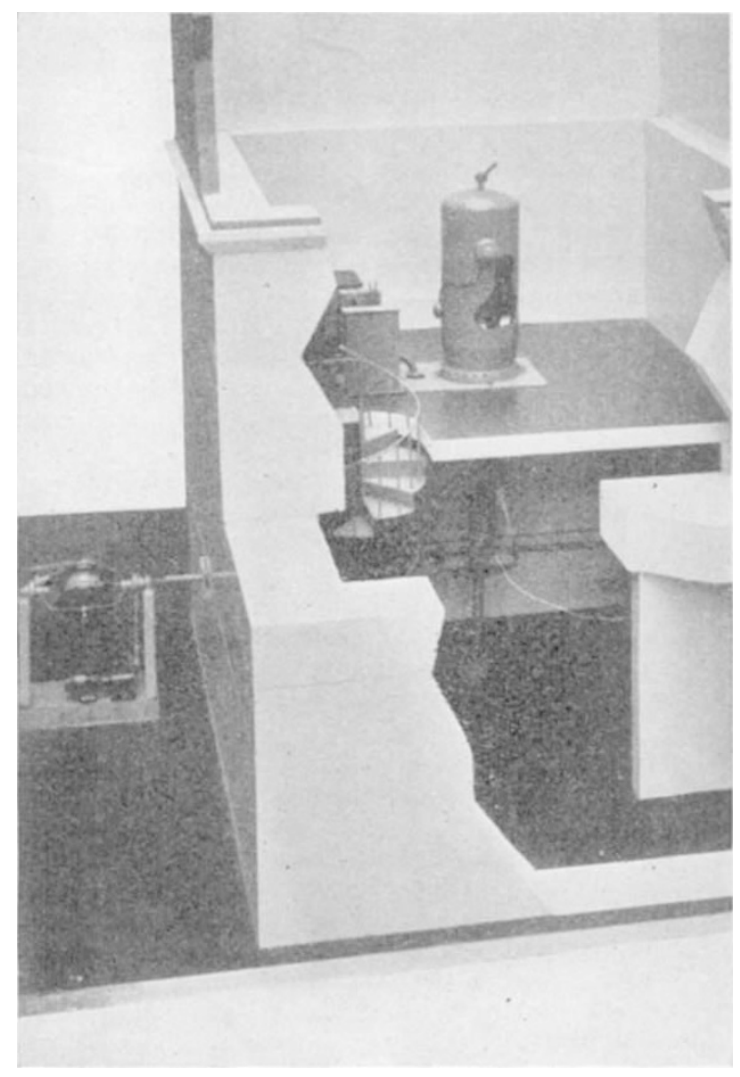

Fig. 1. Model of irradiation block, showing scanning assembly 


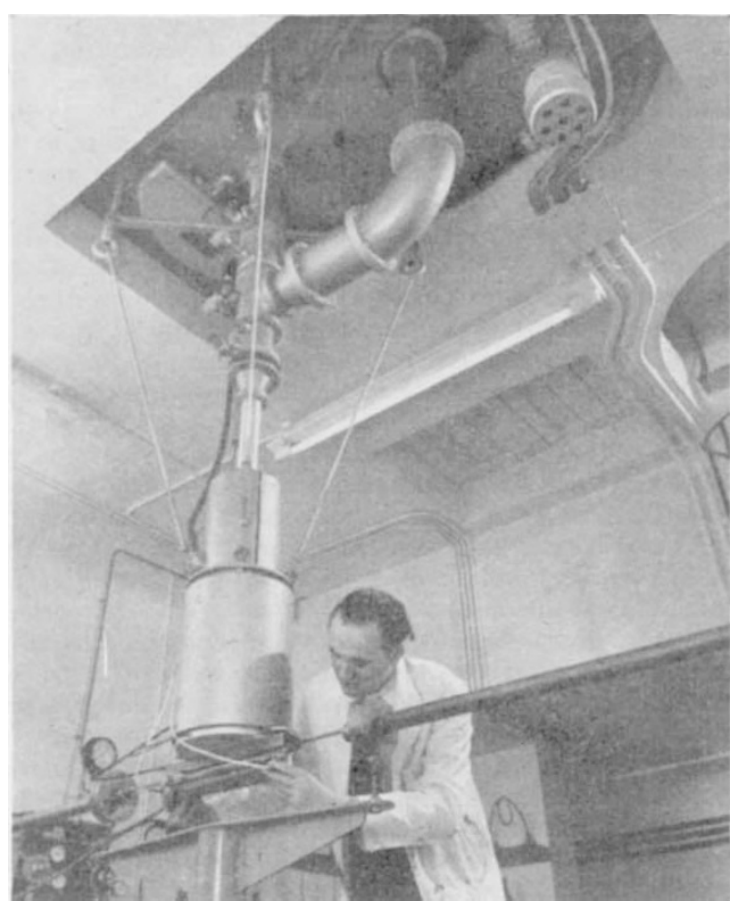

Fig. 2. Scanning unit, showing the base of the generator with diffusion pump and vacuum system

ably lower than in the target room, the thickness of the generator room walls needs to be only $2 \mathrm{ft}$.

The water supply for the laboratories and for the generator is provided by two large tanks situated on the roof of the radiation block, and the necessary pressure for the cooling system of the Van de Graaff accelerator is obtained with boost pumps.

The generator block is flanked on both sides by laboratories which contain the control panels of the generator and various types of cable-handling machinery ; and they are equipped for carrying out the various evaluation tests necessary and investigations upon the irradiated products.

Of the irradiated dielectric materials so far studied, one of the most interesting is polyethylene, as this polymer is cross-linked by high-energy radiation to give a polymer network which has a greatly reduced solubility in hydrocarbon solvents and which exhibits rubber-like elasticity at higher temperatures (greater than $115^{\circ}$ C.) instead of melting. This elastic behaviour and non-flowing property at high temperatures would suggest that irradiated polyethylene has considerable possibilities as a high-temperature dielectric and, while the chemical changes accompanying the cross-linking tend to render the polymer more susceptible to thermal oxidation, investigation has shown that this can be reduced by the prior incorporation of a suitable antioxidant.

Radiation effects such as cross-linking and degradation are not, of course, limited to materials of high molecular weight. Hydrocarbons such as hexane and decane show a similar behaviour, and a study of simple materials is beginning to throw light upon some of the mechanisms of electrical breakdown phenomena. Similarities exist between radiation effects and the ionization effects which sometimes occur in high-voltage equipment under conditions of electrical stress. The well-known phenomenon of waxing or 'cheese' formation in some types of solid cable is only another form of cross-linking by electron bombardment, while the radio-oxidation which takes place during the irradiation of materials in the presence of air bears a direct relationship to the stress-ageing effects encotantered in insulating oils contaminated with oxygen.

High-energy particle accelerators serve not only as a source of radiation for industrial processing but also as valuable laboratory tools for fundamental research. There is no doubt that the new techniques which have arisen during the development of atomic energy in the past ten years will find increasing application in the solving of technical problems which arise in the electrical industry.

\section{PROPERTIES AND USES OF FERRITES CONFERENCE IN LONDON}

$I^{T}$ ' is difficult to-day to name a branch of the sciences or a field of industry or of human endeavour in which electronic devices play no part. During the past twenty-five years the electronics industry throughout the world has expanded at a very great rate, and new discoveries can be of major commercial value. It is not surprising, therefore, that reports ten years ago of the successful preparation of new magnetic materials with obvious applications in electronics and light electrical engineering were received with marked interest.

The use of ferromagnetic materials in coils and transformers had hitherto been restricted to the low-frequency region because of eddy-current losses in the metallic ferromagnetics available. Some improvement had been achieved by the use of fine metallic powders in a non-magnetic matrix-'dust cores'-but while eddy-current losses were thereby reduced, hysteresis losses were usually considerably increased. In 1946, some results were announced of work carried out under great difficulties at the Philips Laboratories in Holland during the German Occupation. The late Dr. J. L. Snoek and his colleagues had succeeded in preparing oxide materials with strong ferromagnetic properties, high electrical resistivity and low hysteresis loss. These materials were ferrites (in fact, mixed crystals of different ferrites) which had first been prepared by Hilpert in 1909 ; but Hilpert's materials, though ferromagnetic, had shown high losses in the presence of alternating magnetic fields and low permeability, and had no commercial value. Snoek's materials had considerable value and were soon made available commercially by Philips under the name of 'Ferroxcube'; later they were manufactured in the United States under the trade name 'Ferramic'.

The engineer was reasonably content, at first, to devote his skill and ingenuity to the use of the available materials, but the physicist and the physical chemist found much that was perplexing, particularly 